NASA Technical Memorandum 106123

$$
\begin{aligned}
& 1 N 57 \\
& 167939
\end{aligned}
$$

\title{
Explicit Robust Schemes for Implementation of General Principal Value-Based Constitutive Models
}

S.M. Arnold

Lewis Research Center

Cleveland, Ohio

and

A.F. Saleeb, H.Q. Tan, and Y. Zhang

University of Akron

Akron, Ohio

May 1993
(NASA-TM-106123) EXPLICIT ROBUST

SCHEMES FOR IMPLEMENTATION OF

GENERAL PRINCIPAL VALUE-BASED

CONSTITUTIVE MODELS (NASA) $29 p$
N93-26947

Unclas

$63 / 59 \quad 0167939$ 


\title{
Explicit Robust Schemes for Implementation of General Principal Value-Based Constitutive Models
}

\author{
S. M. Arnold \\ National Aeronautics and Space Administration \\ Lewis Research Center \\ Cleveland, Ohio 44135
A. F. Saleeb, H. Q. Tan, and Y. Zhang
University of Akron
Akron, Ohio 44325

\begin{abstract}
The issue of developing effective and robust schemes to implement general hyperelastic constitutive models is addressed. To this end, special purpose functions are nsed to symbolically derive, evaluate, and automatically generate the associated FORTRAN code for the explicit forms of the corresponding stress function and material tangent stiffness tensors. These explicit forms are valid for the entire deformation range (i.e.,with both distinct and repeated principal-stretch values). The analytical form of these explicit expressions is given here for the case in which the strainenergy potential $W$ is taken as a nonseparable polynomial function of the principle stretches.
\end{abstract}

\section{Introduction}

Recently, constitutive models of rubber hyperelasticity, using alternative representations in terms of the principal stretches as opposed to deformation invariants, have become increasingly popular in nonlinear finite element analyses [1-3]. Two of our recent publications have discussed in detail the theoretical development [4] and symbolic and numeric implementation [5] of explicit forms for the second Piola Kirchhoff stress tensor and the material tangent stiffness tensor. These forms correspond to a class of Ogden type hyperelastic constitutive models, based on principal-stretch values. These explicit expressions are 
significant since they are valid for the entire deformation range, even though the main constituents of the deformation tensor (i.e., principle values and associated eigenvectors) are, in general, neither uniquely defined nor continuously differentiable over the entire range. The two specific forms of the Ogden-type strain energy functions addressed in reference 4 encompass many of the popular representations currently in use for rubber materials. However, those functions were restricted to special forms of nonseparable representations of the strain energy density functions, with the restricted nonseparable form given in reference 4 , section 5, dealing with the important and practical case of incompressible and slightly compressible solids. To date, comparable treatments for the general nonseparable forms of the models are not available in the literature. Indeed, it is the extension of our earlier results [4] and recent developments [5] to deal with this latter case that constitutes our main objective in the present paper.

By cleverly applying symbolic manipulation packages so as to control expression growth new constitutive theories can be developed and applied (e.g., finite element; see, [6] and [7]). Symbolic computation uses numbers, formulas, vectors, matrices, equations and the like to compute exact solutions; whereas numerical computation uses floating-point numbers to compute approximate solutions to problems of practical interest. Here, we will utilize three recently developed [5] special purpose symbolic functions (SDIFF, SDIFFEV, and TEMPLATE) running under DOE MACSYMA [8]. These special purpose functions allow the derivation and automatic FORTRAN code generation of alternative generalized potential based constitutive models composed of principal values and their associated eigenvectors.

This paper begins by reviewing highlights of our previous work in developing the theory of explicit forms [4] and implementing them symbolically and numerically [5]. Following this review, the results of the derivation of the generalized expressions for the second Piola Kirchhoft stress tensor $S_{i j}$ and the material moduli tensor $D_{i j k l}$ are given. The paper then concludes with a discussion of the template files required to automatically generate the associated FORTRAN source code.

\section{Background}

The theoretical development of a singularity-free representation of principal value-based constitutive models has been discussed at length in reference [4]. Here, we will confine our discussion to hyperelastic isotropic materials whose strain energy function $W$ is taken to be a general function of the principal stretches, that is,

$$
W=W\left(\lambda_{1}, \lambda_{2}, \lambda_{3}\right)
$$

where $\lambda_{1}, \lambda_{2}, \lambda_{3}$ are the principal values of the right Cauchy-Green deformation tensor $C_{i j}$. Denoting $n_{i}(i=1,2,3)$ to be the associated eigenvectors of $C_{i j}$, we 
can define,

$$
C_{i j}=\sum_{l=1}^{3} \lambda_{(l)} N_{i j}^{(l)}
$$

where $N_{i j}^{(l)}$, which is often referred to as the (orthogonal) eigenprojection operator related to the associated eigenvectors of $C_{i j}$ is defined as

$$
N_{i j}^{(l)}=n_{i}^{l} n_{j}^{l}
$$

Equation (2) is valid when all three eigenvalues $\left(\lambda_{i}\right)$ are distinct. However, when two eigenvalues are the same (i.e, double coalescence, $\lambda_{1} \neq \lambda_{2}=\lambda_{3}=\lambda$ ), we have

$$
C_{i j}=\left(\lambda_{1}-\lambda\right) N_{i j}^{(1)}+\lambda \delta_{i j}
$$

And for the case of triple coalescence $\left(\lambda_{1}=\lambda_{2}=\lambda_{3}=\lambda\right)$, we have

$$
C_{i j}=\lambda \sum_{l=1}^{3} N_{i j}^{(l)}=\lambda \delta_{i j} .
$$

Similarly, through suitable manipulation of equations (2) and (4), explicit expressions for $N_{i j}^{(r)}$ in terms of $C_{i j}$ can be obtained for the case of three distinct eigenvalues,

$$
N_{i j}^{(r)}=\frac{1}{\left(\lambda_{r}-\lambda_{s}\right)\left(\lambda_{s}-\lambda_{t}\right)}\left[\left(C_{i j}-\lambda_{s} \delta_{i j}\right)\left(C_{i j}-\lambda_{t} \delta_{i j}\right)\right]
$$

and for the case of double coalescence,

$$
N_{i j}^{(r)}=\frac{1}{\left(\lambda_{r}-\lambda\right)}\left(C_{i j}-\lambda \delta_{i j}\right)
$$

In the preceding equations $\mathrm{r}, \mathrm{s}$, and $\mathrm{t}$ represent any cyclic permutation of $(1,2$, or 3 ). These definitions will prove very useful in obtaining the pertinent singularityfree directional derivatives of both the potential $W$ and the stress function $S_{i j}=S_{i j}\left(C_{i j}\right)$.

The explicit singularity-free expressions for the second Piola Kirchhoff stress tensor $S_{i j}\left(C_{i j}\right)$ are defined as

$$
S_{i j}=2 \frac{\partial W}{\partial C_{i j}} \equiv S_{i j}\left(C_{i j}\right)
$$

Those for the material moduli tensor $D_{i j k l}\left(C_{i j}\right)$ can then be obtained by applying the directional derivative formula to $S_{i j}$, that is,

$$
D_{i j k l}=2 \frac{\partial S_{i j}}{\partial C_{k l}}=4 \frac{\partial^{2} W}{\partial C_{i j} \partial C_{k l}} \equiv D_{i j k l}\left(C_{i j}\right)
$$


As a result, the explicit expressions for the tensors $S_{i j}\left(C_{i j}\right)$ and $D_{i j k l}\left(C_{i j}\right)$ can be obtained directly for the following three cases: case I - when all three eigenvalues are distinct; case II - when a single singularity is present $\left(\lambda_{1} \neq\right.$ $\lambda_{2}=\lambda_{3}=\lambda$, i.e., double coalescence); or case III - when a double singularity is present $\left(\lambda_{1} \neq \lambda_{2}=\lambda_{3}=\lambda\right.$, i.e., triple coalescence).

The derivation and implementation process for the formulations described was recently automated [5] by constructing three special purpose functions (SDIFF, SDIFFEV, and TEMPLATE), written at the MACSYMA command level, that can respectively,

(1) Derive the explicit expressions for the stress tensor $S_{i j}$ (eqs. (8)) and material tensor $D_{i j k l}$ (eqs.(9)), given three, one or no distinct eigenvalues

(2) Evaluate symbolically the expressions generated by SDIFF for a given strain-energy function $\mathrm{W}$

(3) Evaluate the expressions generated by SDIFF and automatically generate (using the built-in MACSYMA function gentran) the associated FORTRAN code needed to evaluate the expressions numerically for a given potential function, $W$

These three special purpose functions contain a list of built-in MACSYMA instructions (factor, expand, ev, ratsubst, diff, limit and for-loops, to name a few) arranged in a specific algorithmic order. Thus each special purpose function can be thought of as a macro command.

\section{Symbolic Derivation}

Let us begin by assuming that $W$ is a nonseparable function of $\lambda_{1}, \lambda_{2}$, and $\lambda_{3}$. For example,

$$
W=\sum_{n=1}^{p}\left[x_{n}\left(\lambda_{1}+\lambda_{2}+\lambda_{3}\right)^{\alpha_{n}}+y_{n}\left(\lambda_{1} \lambda_{2}+\lambda_{2} \lambda_{3}+\lambda_{3} \lambda_{1}\right)^{\beta_{n}}+z_{n}\left(\lambda_{1} \lambda_{2} \lambda_{3}\right)^{\gamma_{n}}\right]
$$

As a result, when the special purpose function SDIFF is invoked, the scalar derivative of $\mathrm{W}$ with respect to each eigenvalue will no longer be a function of that eigenvalue only, as discussed in reference 5 , but will instead be a function of all three eigenvalues, that is,

$$
s_{(l)}\left(\lambda_{1}, \lambda_{2}, \lambda_{3}\right)=2 \frac{\partial W}{\partial \lambda_{(l)}} .
$$

Furthermore, in deriving $D_{i j k l}$, the mixed second derivatives $\left(\frac{\partial^{2} W}{\partial \lambda_{i} \lambda_{j}}\right)$ must also be taken into account in the procedure. To derive the generalized explicit expression for the three cases, one need only issue the command SDIFF upon invoking MACSYMA, as shown here: 
- Case I -three distinct eigenvalues $\left(\lambda_{1} \neq \lambda_{2} \neq \lambda_{3}\right)$

$S D I F F(1)$

- Case II - double coalescence $\left(\lambda_{1} \neq \lambda_{2}=\lambda_{3}=\lambda\right)$

$S D I F F(2)$

- Case III - triple coalescence $\left(\lambda_{1}=\lambda_{2}=\lambda_{3}=\lambda\right)$

$$
S D I F F(3)
$$

Note that the resulting derived expressions have been manipulated and condensed so as to streamline their reporting and to facilitate their comparison with previous work [4].

\subsection{Results for Case I}

The explicit expression for the second Piola Kirchhoff stress tensor is

$$
S_{i j}=a C_{i k} C_{k j}+b C_{i j}+c \delta_{i j}
$$

where $\delta_{i j}$ is the second order identity tensor and a,b, and $c$ are defined as

$$
\begin{gathered}
a=-m\left[s_{1}\left(\lambda_{2}-\lambda_{3}\right)+s_{2}\left(\lambda_{3}-\lambda_{1}\right)+s_{3}\left(\lambda_{1}-\lambda_{2}\right)\right] \\
b=m\left[s_{1}\left(\lambda_{2}^{2}-\lambda_{3}^{2}\right)+s_{2}\left(\lambda_{3}^{2}-\lambda_{1}^{2}\right)+s_{3}\left(\lambda_{1}^{2}-\lambda_{2}^{2}\right)\right] \\
c=-m\left[s_{1} \lambda_{2} \lambda_{3}\left(\lambda_{2}-\lambda_{3}\right)+s_{2} \lambda_{3} \lambda_{1}\left(\lambda_{3}-\lambda_{1}\right)+s_{3} \lambda_{1} \lambda_{2}\left(\lambda_{1}-\lambda_{2}\right)\right]
\end{gathered}
$$

and where

$$
m=\frac{1}{\left(\lambda_{1}-\lambda_{2}\right)\left(\lambda_{2}-\lambda_{3}\right)\left(\lambda_{3}-\lambda_{1}\right)}
$$

The explicit expression for the material moduli tensor $D_{i j k l}\left(C_{i j}\right)$ is

$$
\begin{gathered}
D_{i j k l}=a_{1} P\left(C_{k l}^{2}, C_{i j}^{2}\right)+a_{2}\left[P\left(C_{k l}^{2}, C_{i j}\right)+P\left(C_{k l}, C_{i j}^{2}\right)\right] \\
+a_{3}\left[Q\left(C_{k l}^{2} \delta_{i j}\right)+P\left(\delta_{k l}, C_{i j}^{2}\right)\right]+a_{4} P\left(C_{k l}, C_{i j}\right) \\
+a_{5}\left[Q\left(C_{k l}, \delta_{i j}\right)+Q\left(\delta_{k l}, C_{i j}\right)\right]+2 a_{6} I_{i j k l}
\end{gathered}
$$

where two second order symmetric tensors $P$ and $Q$ have been introduced and are defined as

$$
P_{i j k l}(G, H)=G_{i k} H_{j l}+G_{i l} H_{j k}
$$




$$
Q_{i j k l}(G, H)=G_{i k} H_{j l}+G_{i j} H_{j k}+G_{j l} H_{i k}+G_{j k} H_{i l}
$$

and the notation

$$
\begin{gathered}
I_{i j k l}=\frac{1}{2}\left[\delta_{i k} \delta_{j l}+\delta_{i l} \delta_{j k}\right] \\
C_{i j}^{2}=C_{i m} C_{m j}
\end{gathered}
$$

has been used in equation (15). Here the coefficients $a_{1}, a_{2}, \ldots, a_{6}$ are defined as

$$
\begin{gathered}
a_{1}=\sum_{r=1}^{3} \eta_{r}+\sum_{r=1}^{3} \sum_{s=1, r \neq s}^{3} \xi_{r s} \\
a_{2}=\sum_{r=1}^{3}\left(\lambda_{r}-I_{1}\right) \eta_{r}-\frac{1}{2} \sum_{r=1}^{3} \sum_{s=1, r \neq s}^{3}\left(2 I_{1}-\lambda_{r}-\lambda_{s}\right) \xi_{r s} \\
a_{3}=\sum_{r=1}^{3} \frac{I_{3} \eta_{r}}{\lambda_{r}}+\frac{1}{2} \sum_{r=1}^{3} \sum_{s=1, r \neq s}^{3}\left(\lambda_{r}+\lambda_{s}\right)\left(I_{1}-\lambda_{r}-\lambda_{s}\right) \xi_{r s} \\
a_{4}=\sum_{r=1}^{3}\left(I_{1}-\lambda_{r}\right)^{2} \eta_{r}+\frac{1}{2} \sum_{r=1}^{3} \sum_{s=1, r \neq s}^{3}\left(I_{1}-\lambda_{r}\right)\left(I_{1}-\lambda_{s}\right) \xi_{r s} \\
a_{5}=\sum_{r=1}^{3}\left(\mu_{r}+\frac{I_{3} \eta_{r}\left(\lambda_{r}-I_{1}\right)}{\lambda_{r}}\right)-\frac{1}{2} \sum_{r=1}^{3} \sum_{s=1, r \neq s}^{3}\left(I_{2}+\lambda_{r} \lambda_{s}\right)\left(I_{1}-\lambda_{r}-\lambda_{s}\right) \xi_{r s} \\
a_{6}=\sum_{r=1}^{3}\left(\frac{I_{3}}{\lambda_{r}}\right)^{2} \eta_{r}+\left(\lambda_{r}-I_{1}\right) \mu_{r}+\sum_{r=1}^{3} \sum_{s=1, r \neq s}^{3} I_{3}\left(I_{1}-\lambda_{r}-\lambda_{s}\right) \xi_{r s}
\end{gathered}
$$

where

$$
\begin{gathered}
\mu_{r}=\frac{s_{r}}{\left(\lambda_{r}-\lambda_{s}\right)\left(\lambda_{r}-\lambda_{t}\right)} \\
\eta_{r}=\frac{\left[s_{r r}+\left(\lambda_{s}-\lambda_{r}\right)\left(\mu_{r}+\mu_{t}\right)+\left(\lambda_{t}-\lambda_{r}\right)\left(\mu_{r}+\mu_{s}\right)\right]}{\left(\lambda_{r}-\lambda_{s}\right)^{2}\left(\lambda_{r}-\lambda_{t}\right)^{2}} \\
\xi_{r s}=\frac{s_{r s}}{\left(\lambda_{r}-\lambda_{s}\right)^{2}\left(\lambda_{r}-\lambda_{t}\right)\left(\lambda_{s}-\lambda_{t}\right)}
\end{gathered}
$$

and

$$
\begin{gathered}
I_{1}=\lambda_{1}+\lambda_{2}+\lambda_{3} \\
I_{2}=\lambda_{1} \lambda_{2}+\lambda_{2} \lambda_{3}+\lambda_{1} \lambda_{3} \\
I_{3}=\lambda_{1} \lambda_{2} \lambda_{3}
\end{gathered}
$$

Note that in the preceding expression the following differentiation notation has been introduced: 


$$
s_{l}=s_{l}\left(\lambda_{1}, \lambda_{2}, \lambda_{3}\right)=2 \frac{\partial W}{\partial \lambda_{(l)}}
$$

and

$$
s_{i j}=s_{i j}\left(\lambda_{1}, \lambda_{2}, \lambda_{3}\right)=\frac{\partial s_{i}\left(\lambda_{1}, \lambda_{2}, \lambda_{3}\right)}{\partial \lambda_{j}}=\frac{\partial^{2} W}{\partial \lambda_{i} \lambda_{j}}
$$

For example, $s_{11}=2 \frac{\partial^{2} W}{\partial \lambda_{1}^{2}} ; s_{22}=2 \frac{\partial^{2} W}{\partial \lambda_{2}^{2}} ; s_{33}=2 \frac{\partial^{2} W}{\partial \lambda_{3}^{2}} ; s_{12}=s_{21}=\frac{\partial^{2} W}{\partial \lambda_{1} \lambda_{2}} ; s_{13}=$ $s_{31}=\frac{\partial^{2} W}{\partial \lambda_{1} \lambda_{1}} ; s_{23}=s_{32}=\frac{\partial^{2} W}{\partial \lambda_{2} \lambda_{3}}$. A comparison of the preceding expressions and those obtained earlier for the two special Ogden-type strain energy forms [4], shows that the expressions are identical except for the additional doublesummation terms (containing the cross derivative terms) in the coefficients $a_{1}, a_{2}, \ldots a_{6}$ (see equations (20)-(25)) comprising the material moduli tensor $D_{i j k l}$. Thus the previous work is now merely a special case of the present generalized expressions.

\subsection{Results for Case II}

In this case, a single singularity $\left(\lambda_{1} \neq \lambda_{2}=\lambda_{3}=\lambda\right)$ is analytically removed, thereby yielding

$$
S_{i j}=\bar{a} C_{i j}+\bar{b} \delta_{i j}
$$

with

$$
\begin{gathered}
\bar{a}=\frac{s_{1}-s_{2}}{\left(\lambda_{1}-\lambda\right)} \\
\bar{b}=-\frac{\left[s_{1} \lambda-s_{2} \lambda_{1}\right]}{\left(\lambda_{1}-\lambda\right)}
\end{gathered}
$$

and a reduced material moduli tensor

$$
D_{i j k l}=b_{1} P\left(C_{k l}, C_{i j}\right)+b_{2}\left[Q\left(C_{k l}, \delta_{i j}\right)+Q\left(\delta_{k l}, C_{i j}\right)\right]+b_{3} I_{i j k l}
$$

where

$$
\begin{gathered}
b_{1}=\frac{1}{\left(\lambda_{1}-\lambda_{2}\right)^{3}}\left\{\left(\lambda_{1}-\lambda_{2}\right)\left[s_{11}+s_{22}-2 s_{12}\right]-2\left[s_{1}-s_{2}\right]\right\} \\
b_{2}=\frac{1}{\left(\lambda_{1}-\lambda_{2}\right)^{3}}\left\{\left(\lambda_{2}-\lambda_{1}\right)\left[\lambda_{2} s_{11}+\lambda_{1} s_{22}-2\left(\lambda_{1}+\lambda_{2}\right) s_{12}\right]+\left(\lambda_{1}+\lambda_{2}\right)\left[s_{1}-s_{2}\right]\right\} \\
b_{3}=\frac{1}{\left(\lambda_{1}-\lambda_{2}\right)^{3}}\left\{\left(\lambda_{1}-\lambda_{2}\right)\left[\lambda_{1}^{2} s_{22}+\lambda_{2}^{2} s_{11}-2 \lambda_{1} \lambda_{2} s_{12}\right]-2 \lambda_{1} \lambda_{2}\left[s_{1}-s_{2}\right]\right\}
\end{gathered}
$$

Again, in comparing the coefficients a and $\mathrm{b}$, and $b_{1}, b_{2}$, and $b_{3}$ to those obtained in previous work [4], the only difference seen is the appearance of the cross derivative term $\left(s_{12}\right)$ in coefficients $b_{1}, b_{2}$, and $b_{3}$. 


\subsection{Results for Case III}

Finally, in the case of a double singularity $\left(\lambda_{1}=\lambda_{2}=\lambda_{3}=\lambda\right)$, the explicit expression for the stress tensor becomes

$$
S_{i j}=s_{\lambda}(\lambda) \delta_{i j}
$$

whereas the material moduli tensor becomes

$$
D_{i j k l}=2 s_{\lambda \lambda}(\lambda) \delta_{i j k l} .
$$

These are identical to the previous results, as one would expect.

The value of automating the foregoing derivation procedure is apparent in that not only does this special purpose function SDIFF relieve the user of the tedious manual derivation process, but it also ensures analytical accuracy. This was illustrated prior to the publication of reference 4 , in that a number of errors in the hand derivation were detected, verified, and corrected. Also, because the derivation process needs to be executed only once, except for the evaluation of the scalar derivatives in equations (26) and (27) for each new definition of $W$, a second special purpose function, SDIFFEV, as described in [5], was developed. This function is used to symbolically evaluate the foregoing expressions.

\subsection{FORTRAN Code Generation}

The function TEMPLATE is similar to the function SDIFFEV in that both functions will evaluate the explicit expressions obtained from SDIFF. TEMPLATE, however, will automatically generate the associated FORTRAN source code needed to numerically evaluate the expressions for a given potential function W. Code generation is accomplished by utilizing the MACSYMA built-in function gentran and a number of template files. The template files can be thought of as a framework for the FORTRAN generation of four subroutines (the main driving routine COMPSD and three subroutines, one each for case I, case II, and case III) and numerous functions. The template file for the main driving routine COMPSD is shown in appendix $A$. This subroutine is constructed for easy implementation into a finite element code. The input requirements are the strain tensor $C_{m}$ (denoted as $\mathrm{cmu}$ ) and its associated eigenvalues $\lambda_{1}, \lambda_{2}$, and $\lambda_{3}$ (denoted by gl1, gl2, and gl3 respectively). The outputs are the stress tensor $S_{n}$ (denoted as s) and the material moduli tensor $D_{n m}$ (denoted as d). Here, $n$ and $m$ run from 1 to 6 . Clearly, the only code generation required is that of subroutines COMPSD1, COMPSD2, and COMPSD3. Code generation is initiated by issuing the command gentranin, preceded by and followed by less than and greater than symbols, respectively.

The subroutines COMPSD1, COMPSD2, and COMPSD3 are associated with case I $\left(\lambda_{1} \neq \lambda_{2} \neq \lambda_{3}\right)$, case II $\left(\lambda_{1}, \lambda=\lambda_{2}=\lambda_{3}\right)$, and case III $(\lambda=$ $\lambda_{1}=\lambda_{2}=\lambda_{3}$ ), described in section 2.0. The template files corresponding to 
these three cases are shown in appendices $B, C$ and $D$, respectively. Note that in these routines, most of the FORTRAN code is automatically generated, since it pertains to the definition of coefficients $a, b, c ; a_{1}, a_{2}, \ldots, a_{6}$, and the first and second scalar derivatives of the strain energy function W, (i.e., $s_{1}, s_{2}, s_{3}, s_{11}, s_{22}$, and $\left.s_{33}\right)$. Also, the gentran commands are again preceded and followed by double inequality signs (that is, $\ll \gg$ ). All functions that are associated with a given case have been included in the corresponding appendix. As a result, with the appropriate template files, the FORTRAN source code associated with any general nonseparable or separable strain-energy function can easily be generated.

\section{Summary of Results}

Taken separately, the main constituents of the deformation tensor (i.e., the principal values and associated eigenvectors) are, in general, not uniquely defined and continuously differentiable functions. Careful consideration is thus called for in implementing constitutive models formulated in terms of these principalstrain measures. This difficulty was entirely bypassed by resorting to explicit symbolic derivations of appropriate forms of the material tangent-stiffness matrices which are valid for the entire deformation range. Furthermore, to enhance effective utilization and implementation of the present results, automatic FORTRAN code generation of the present generalized explicit expressions was pursued and achieved. As a result, nonseparable forms dealing with the important practical case of incompressible and slightly compressible solids can easily be generated. Finally, the generic analytical forms of these explicit expressions have been given for three cases: (1) distinct eigenvalues, (2) one distinct eigenvalue, and (3) no distinct eigenvalues.

In the future we will broaden our scope of application to include not only deformation constitutive models but also damage representations as well. An example that immediately comes to mind, where the above singularity-free representations will be important, is a maximum principle stress (or strain) damage formulation. Using this work as a building block, we can then envision moving to even more sophisticated damage formulations involving even higher tensorial representations.

\section{References}

[1] Finney, R.H.; and Kumar, A.: Development of Material Constants for Nonlinear Finite Element Analysis. J. Rubber Chem. Tech., Vol.. 61, pp. 879 $891,1988$.

[2] Sussman, T.; and Bathe, K.J.: A Finite Element Formulation for Nonlinear Incompressible Elastic and Inelastic Analysis. Comp. Struct., Vol. 26, pp. 357-409, 1987. 
[3] Chang, T.Y.; Saleeb, A.F.; and Li, G.: Large Strain Analysis of Rubber-Like Materials by a Mixed Finite Element. Computational Mech., Vol. 8, No.4, pp. 221-233, 1991.

[4] Saleeb.A.F.; and Arnold, S.M.: Explicit Robust Schemes for Implementation of a Class Of Principle Value-Based Constitutive Models: Theoretical Development. NASA TM 105345, 1991.

[5] Arnold, S.M.; et al.: Explicit Robust Schemes for Implementation of a Class of Principal Value-Based Constitutive Models: Symbolic and Numeric Implementation. NASA TM 106124, 1993.

[6] Arnold, S.M.; and Tan, H.Q.: Symbolic Derivation of Potential Based Constitutive Equations. Computational Mech., Vol. 6, pp. 237-246, 1990.

[7] Arnold, S.M.; Tan, H.Q.; and Dong, X.: Application of Symbolic Computations to The Constitutive Modeling of Structural Materials. Symbolic Computations and Their Impact on Mechanics, Noor, A.K., Elishakoff, I. and Hulbert, G., eds., PVP-Vol. 205, ASME, pp.215-229.,1990.

[8] MATHLAB Group: MACSYMA Reference Manual. Version 10. Laboratory for Computer Science, Massachusetts Institute of Technology, Cambridge, MA, 1984. 
APPENDIX A: Template File Associated With COMPSD

The Main Driver Routine

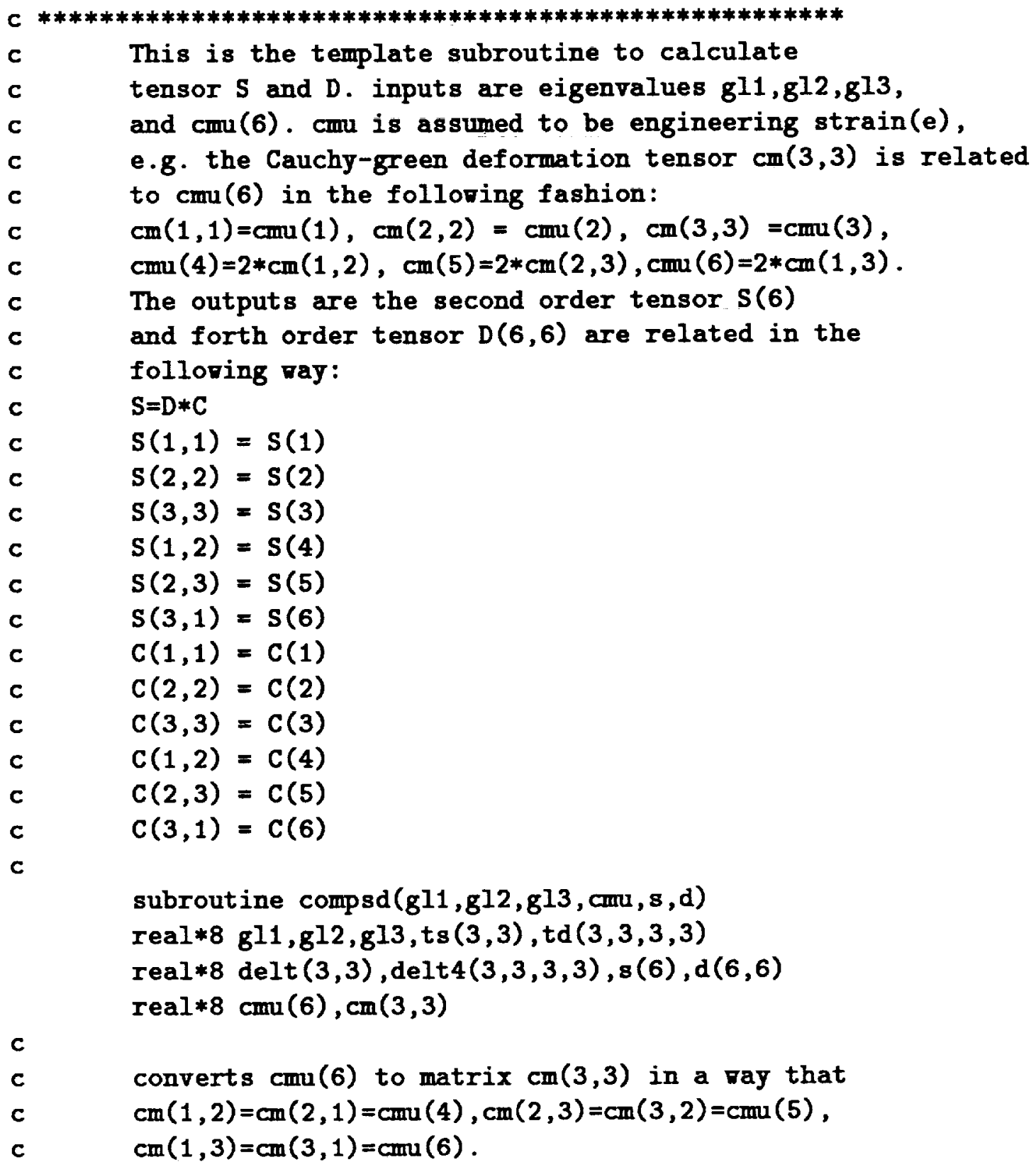


do $5 i=1,3$

do $5 j=1,3$

if $(i . e q \cdot j)$ then

iq $=i$

$\operatorname{cm}(i, j)=\operatorname{cmu}(i q)$

else if (i.ne.j) then

if $((i+j) \cdot e q \cdot 3)$ iq $=4$

if $((i+j) . e q \cdot 4)$ iq=6

if $((i+j)$ eq $\cdot 5)$ iq $=5$

$\operatorname{cm}(i, j)=\operatorname{cmu}(i q) / 2$

end if

continue

5 continue

c

c

c

c

6

c

c

c

c

7

c

$c * * * * * * * * * * * * * * * * * * * * * * * * * * * * * * * * * * * * * * * * * * * * * * * * * * * * * * * * * * * * * * * * * * *$

c For different eigenvalues $\mathrm{gl1}, \mathrm{gl2}, \mathrm{g} 13$ the computation

c is different. case1 is gl1\#gl2\#gl3 call subroutine comsd1.

c case 2 is $\mathrm{g} 13=\mathrm{g} 12 \# \mathrm{~g} 11$ or $\mathrm{g} 11=\mathrm{g} 13 \# \mathrm{~g} 12$ or $\mathrm{g} 11=\mathrm{g} 12 \# \mathrm{~g} 13$ then

c call subroutine compsd2 . case 3 is $g 11=g 12=g 13$ call subroutine

C compsd3.

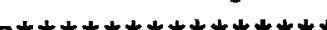

if ((g11.ne.g12).and. (g12.ne.g13).and. (gl1.ne.gl3)) then

call compsd1 (gl1, g12, gl3, delt, delt $4, \mathrm{~cm}, \mathrm{ts}, \mathrm{td})$

else if ( (g12.eq.g13). and. (g11.ne.g13)) then 
call compsd2 (gl1, gl2, delt, delt4, cm,ts, td)

else if ((gl1.eq.gl2). and.(g13.ne.g12)) then

gl1 $=\mathrm{g} 13$

call compsd2 (gl1, gl2, delt, delt $4, \mathrm{~cm}, \mathrm{ts}, \mathrm{td})$

else if ( (gl1.eq.g13).and.(g12.ne.g13)) then

$\mathrm{g} 11=\mathrm{g} 12$

$\mathrm{g} 12=\mathrm{g} 13$

call compsd2 (gl1, gl2, delt, delt $4, \mathrm{~cm}, t \mathrm{~s}, \mathrm{td})$

else

call compsd3 (gl1, delt, delt4,ts,td)

end if

c

c

c

C

c

8

9

10

Rewrite the tensor $t s(i, j) \operatorname{td}(i, j, k, 1)$ to $s(i)$ and $D(i, j)$ respectively by using the symetric property. converts $t s(3,3) s(6)$ and $t d(3,3,3,3)$ to $D(6,6)$

do $8 i=1,3$

do $8 j=i, 3$

if $(i \cdot e q \cdot j)$ iq=i

if (i.eq.1. and.j.eq. 2 ) iq=4

if (i.eq.2. and.j.eq.3) iq=5

if (i.eq.1.and.j.eq.3) iq=6

$s(i q)=t s(i, j)$

continue

continue

do $9 i=1,3$

do $9 j=i, 3$

$d(i, j)=\operatorname{td}(i, i, j, j)$

continue

continue

do $10 i=1,3$

$d(i, 4)=t d(i, i, 1,2)+t d(i, i, 2,1)$

$d(i, 5)=\operatorname{td}(i, i, 2,3)+t d(i, i, 3,2)$

$d(i, 6)=\operatorname{td}(i, i, 3,1)+t d(i, i, 1,3)$

continue

$d(4,4)=(t d(1,2,1,2)+t d(1,2,2,1)+t d(2,1,1,2)+t d(2,1,2,1)) / 2$.

$d(4,5)=(\operatorname{td}(1,2,2,3)+t d(1,2,3,2)+t d(2,1,2,3)+t d(2,1,3,2)) / 2$.

$d(4,6)=(t d(1,2,1,3)+t d(1,2,3,1)+t d(2,1,1,3)+t d(2,1,3,1)) / 2$.

$d(5,5)=(t d(2,3,2,3)+t d(2,3,3,2)+t d(3,2,2,3)+t d(3,2,3,2)) / 2$.

$d(5,6)=(t d(2,3,1,3)+t d(2,3,3,1)+t d(3,2,1,3)+t d(3,2,3,1)) / 2$. 


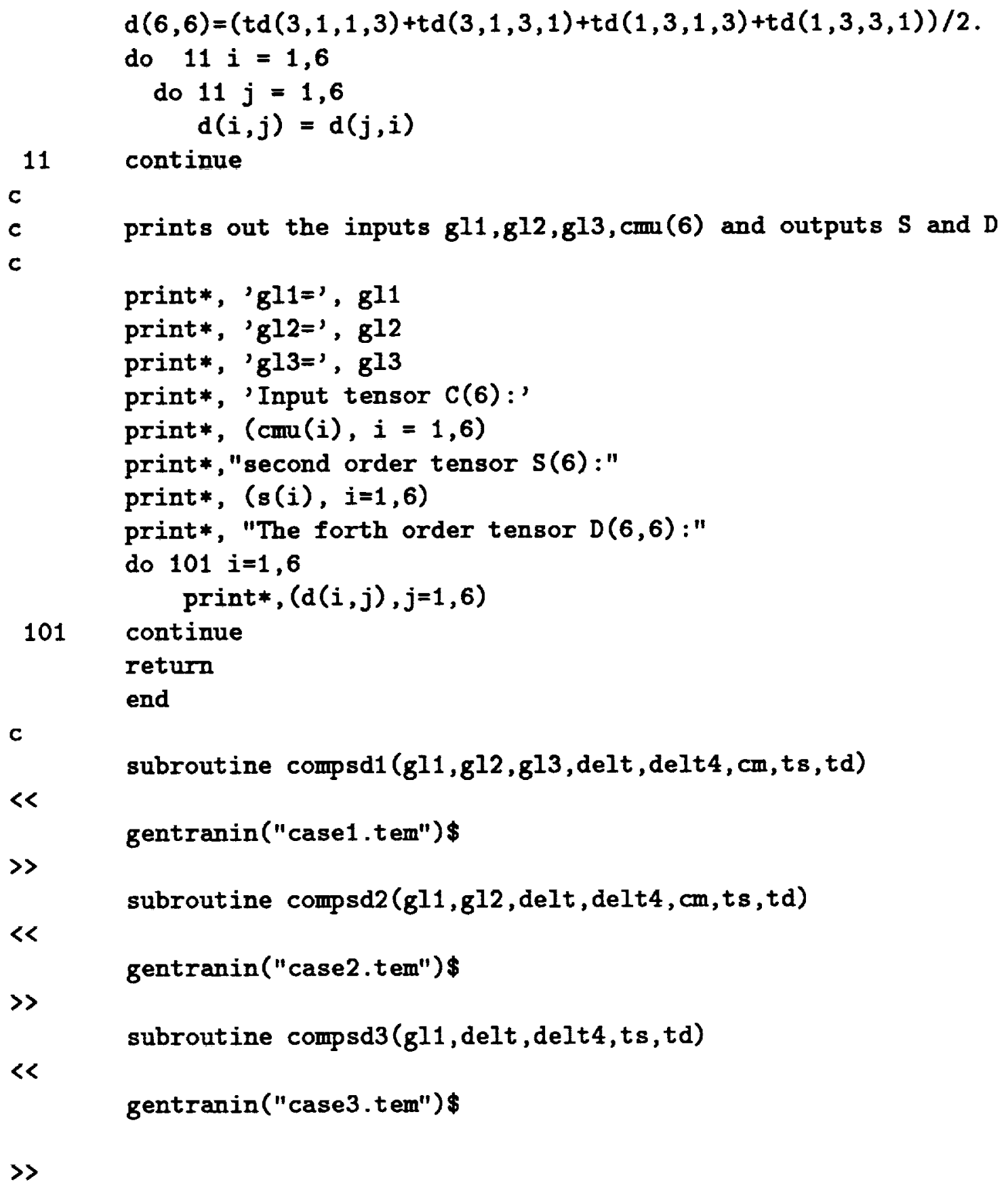


c

c

c

This subroutine computes $P$ and $Q$ forth order tensors

c

c

This subroutine computes matrix product $\mathrm{cmXcm}$.

c

$$
\text { subroutine product (mat 1, cman) }
$$

real $* 8$ mat $1(3,3), \operatorname{cmm}(3,3)$, sum

do $25 i=1,3$

do $25 j=1,3$

sum $=0.0$

do $26 \mathrm{k}=1,3$ sum= sum+mat $1(i, k) * \operatorname{mat} 1(k, j)$

26 continue

25

$$
\operatorname{cmm}(i, j)=\operatorname{sum}
$$

continue

return

end 
APPENDIX B: Template File Associated With COMPSD1

Valid For Three Distinct Eigenvalues

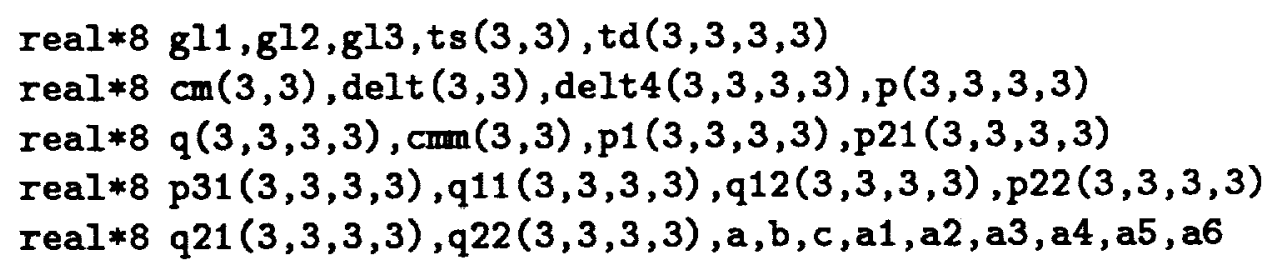

Obtains $\mathrm{cmm}(3,3)=\mathrm{cm}(3,3) * \mathrm{~cm}(3,3)$ from subroutine product

C

call product ( $\mathrm{cm}, \mathrm{cmm})$

c Uses the formula ve derived in code to compute second order

c tensor ts $(3,3)$.

$<$

gentran(for $i: 1$ thru 3 do

(for $j: 1$ thru 3 do

(ts $[i, j]: a(g 11, g 12, g 13) * c \operatorname{mm}[i, j]+b(g 11, g 12, g 13)$ $* c m[i, j]+c(g 11, g 12, g 13) * \operatorname{delt}[i, j]))) \$$

>

Call subroutine to compute all the functions we defined when ve derived forth order tenosor $t d$, namely $P(i, j, k, 1)$ and $Q(i, j, k, 1)$ phich are the functions of $\mathrm{cm}(3,3)$ and the matrix product $\operatorname{cmm}(3,3)$.

call $p q \operatorname{com}(c \mathrm{~mm}, \mathrm{cmm}, \mathrm{p} 1, \mathrm{q})$

call pqcom (cmin, cm, p21, q)

call $p q \operatorname{com}(\mathrm{cm}, \mathrm{cmm}, \mathrm{p} 22, \mathrm{q})$

call pqcom $(\mathrm{cm}, \mathrm{cm}, \mathrm{p} 31, \mathrm{q})$

call pqcom (cmm,delt,p,q11)

call pqcom(delt, comm,,$q 12$ )

call pqcom (cm, delt, p, q21)

call pqcom (delt, cm,p, q22) 


\section{C}

c.

$<<$

$>$

C

C

C

$<<$

>>

$<<$

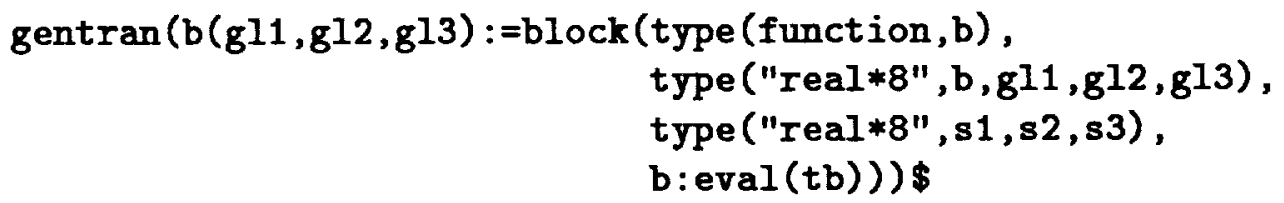

Computes forth order tensor $\operatorname{td}(i, j, k, 1)$

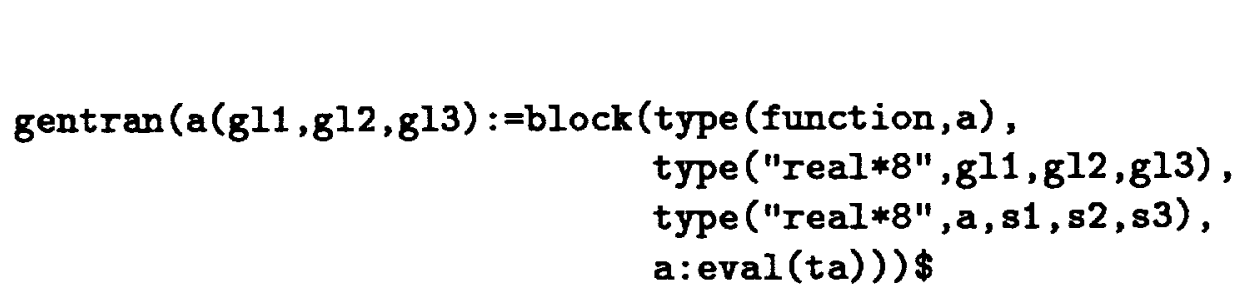

〉 
$<<$

gentran (a1 (gl1, g12,g13):=block(type(function, a1), type ("real*8", a1, gl1, g12,g13), type ("real*8", s1 , s2,s3, s11, s22, s33, s21,s32,s31), a1 : eval (a1)))\$

$>$

$<<$

gentran (a2 (g11, g12, g13): =block(type(function, a2), type ("real*8", a2,g11,g12,g13), type ("real*8", s1,s2,s3,s11, s22,s33,s21, s32,s31), a2 : eval (a2)))\$

$\gg$

$<<$

gentran (a3 (g11, g12, g13):=block(type(function, a3), type ("real*8", a3,g11,g12,g13),

type ("real*8", s1, s2, s3, s11,s22,s33,s21, s32, s31), a3: eval (a3)))\$

$\gg$

$<<$

gent $\operatorname{ran}\left(a 4\left(g 11, g^{12}, g^{13}\right):=b l o c k\right.$ (type (function, a4), type ("real*8", a4,gl1,g12,g13), type ("real*8" , s1, s2 , s3, s11,s22,s33,s21, s32, s31), a4 : eval (a4)))\$

$>$

$<$

gentran (a5 (g11,gl2,gl3):=block(type (function, a5), type ("real*8", a5, g11,g12,g13),

type ("real*8" , s1 , s2 , s3, s11, s22,s33, s21, s32, s31), a5: eval (a5)))\$

$\gg$

$\ll$

gentran $\left(a 6\left(g 11, g^{12}, g^{13}\right):=b l o c k\right.$ (type (function ,a6), type ("real*8" , a6,gl1,g12,g13),

type("real*8", s1, s2,s3,s11,s22,s33,s21,s32,s31), a6:eval (a6)))\$

> 


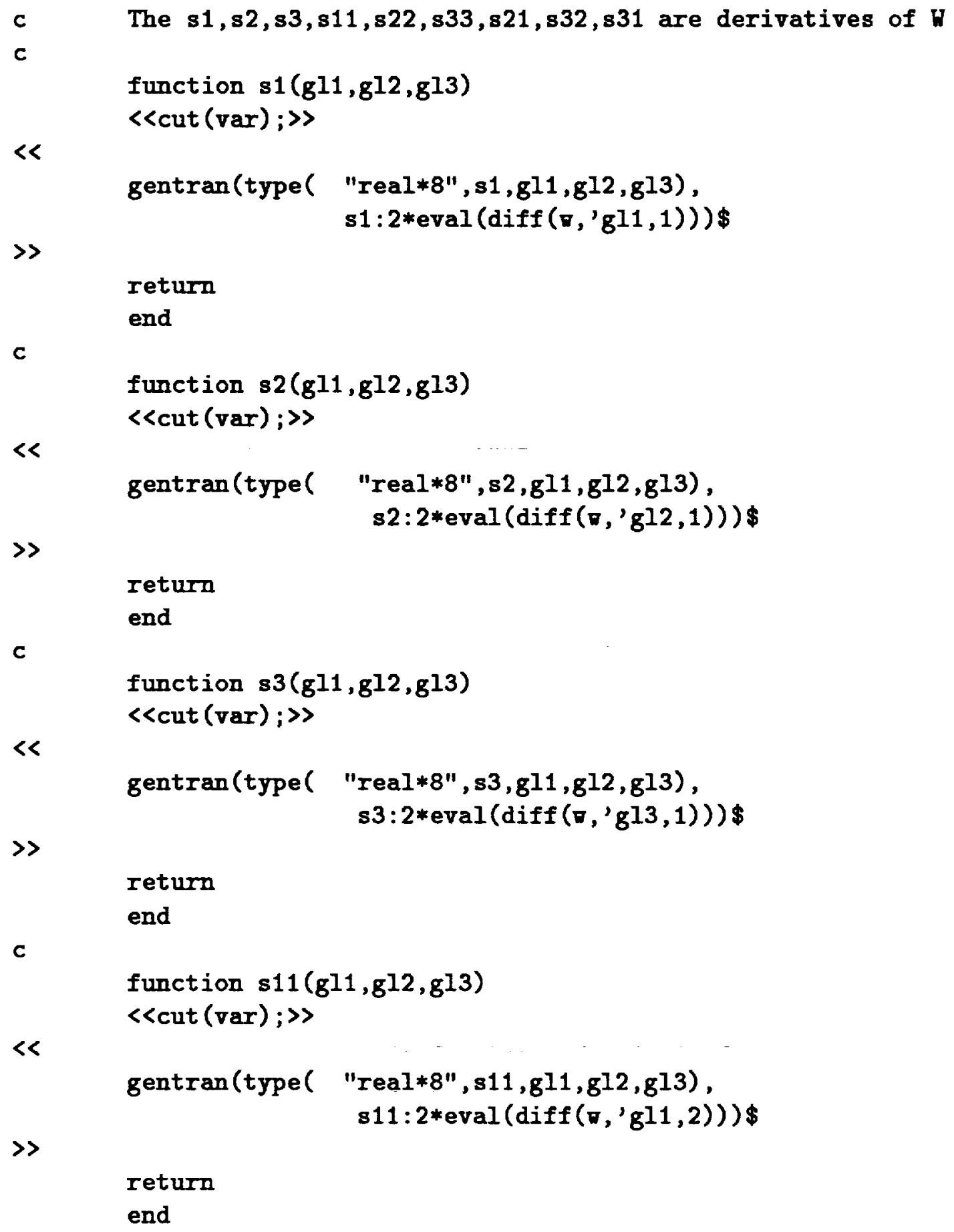

〉

return

end

C

function $s 11\left(\mathrm{~g} 11, \mathrm{~g} 12, \mathrm{~g}^{13}\right)$

<<cut (var); ; >

$<<$

gentran(type( "real*8",s11,g11,g12,g13), $s 11: 2 * \operatorname{eval}(\operatorname{diff}(\nabla, ' g l 1,2))) \$$

〉>

return

end 
c

function $\mathrm{s} 22\left(\mathrm{~g} 11, \mathrm{~g}^{12}, \mathrm{~g} 13\right)$

<<cut (var); >>

$\ll$

gentran (type ("real*8", s22,g11,g12,g13), s22:2*eval (diff $(\pi, ' g 12,2))) \$$

$\gg$

return

end

c

function $\mathrm{s} 33\left(\mathrm{~g} 11, \mathrm{~g}^{12}, \mathrm{~g} 13\right)$

<<cut (var); >>

$<<$

gentran(type( "real*8",s33,g11,g12,g13), s33:2*eval (diff $(\nabla, ' g 13,2))) \$$

$\gg$

return

end

c

function $s 21\left(\mathrm{~g} 11, \mathrm{~g}^{12}, \mathrm{~g} 13\right)$

<<cut (var); ; >

$<$

gentran (type( "real*8", s21,g11,gl2,g13), s21:2*eval (diff $(\square, ' g 12,1, ' g 11,1))) \$$

〉

return

end

c

function $\mathrm{s} 31(\mathrm{~g} 11, \mathrm{~g} 12, \mathrm{~g} 13)$

<<cut (var); >>

$<<$

gentran(type( "real*8",s31,g11,g12,g13), s31:2*eval (diff $(\pi, ' g 13,1, ' g 11,1))) \$$

>

return

end 
c

function $832(\mathrm{~g} 11, \mathrm{~g} 12, \mathrm{~g} 13)$

<<ut (var); ;>

$<$

gentran(type( "real*8", s32,gl1,g12,g13), s32: 2*eval(diff $\left.\left(\pi, g^{\prime}\left(3,1,{ }^{\prime} g 12,1\right)\right)\right) \$$

>>

return

end 
APPENDIX C: Template File Associated With COMPSD2

Valid For Double Coalesence Case

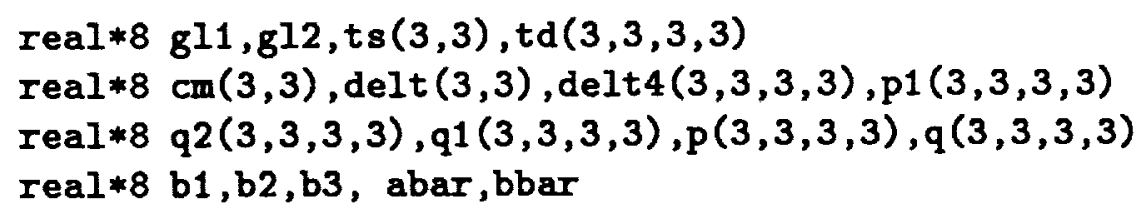

c

$<<$

\>

c

c

c

c

c

c

$<<$

gentran(for $i: 1$ thru 3 do (for $j: 1$ thru 3 do

(for $k: 1$ thru 3 do

(for $1: 1$ thru 3 do (td $[i, j, k, 1]: b 1(g 11, g 12) * p 1[i, j, k, 1]+b 2(g 11, g 12) *$ $(q 1[i, j, k, 1]+q 2[i, j, k, l])+b 3(g 11, g 12) * \operatorname{delt} 4[i, j, k, 1]))))) \$$

gentran(for $i: 1$ thru 3 do

(for $j: 1$ thru 3 do

$(t s[i, j]: a b a r(g 11, g 12) * c m[i, j]+b b a r(g 11, g 12) * \operatorname{delt}[i, j]))) \$$

Call subroutine to get $P, Q$ vhich are defined in code.

call $\mathrm{pqcom}(\mathrm{cm}, \mathrm{cm}, \mathrm{p} 1, \mathrm{q})$

call pqcom (cm, delt,p,q1)

call pqcom(delt, cm, p, q2)

Computes tensor $t d(i, j, k, 1)$.

$\gg$

return

end 


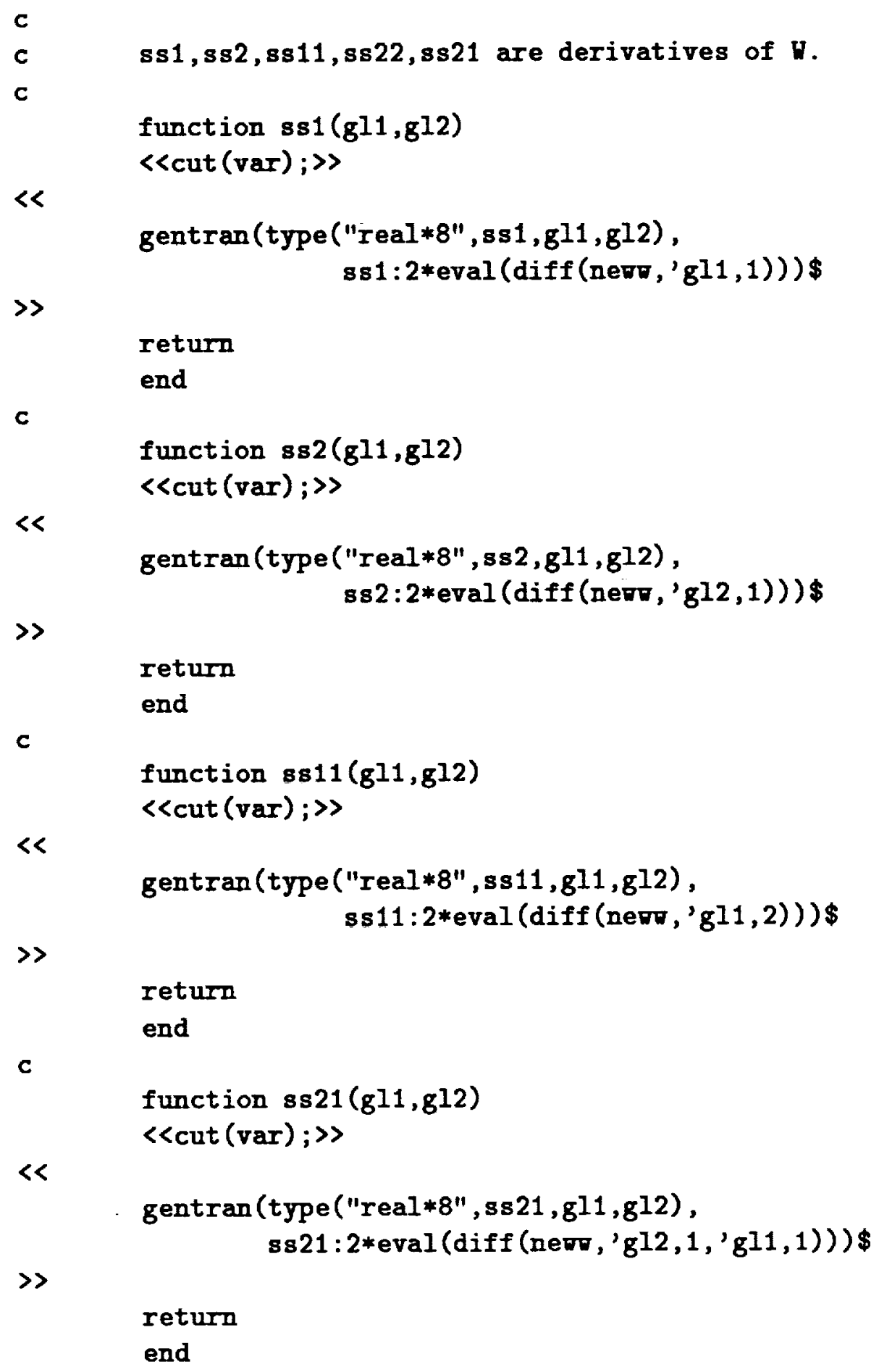

c

function s811 (gl1, g12)

<<cut (var); ; >

$<<$

gentran (type ("real*8", ss11,g11,g12), ss11:2*eval (diff (nerv, ' $g 11,2))$ )\$

>>

return

end

c

function ss21 (g11, gl2)

<<cut (var); >>

$\ll$

gentran (type ("real*8", ss21, g11,g12), ss21:2*eval (diff (newr, 'gl2, 1, 'gl1,1))) \$

>

return

end 
c

function $\mathrm{ss22}(\mathrm{gl1}, \mathrm{g} 12)$

<<cut (var) ; >>

$<<$

gentran (type ("real*8", ss22, gl1,g12), 8822 : $2 * \operatorname{eval}(\operatorname{diff}($ newr, 'gl2, 2$))$ ) \$

〉>

return

end 
APPENDIX D: Template File Associated With COMPSD3

Valid For The Triple Coalesence Case

c

real*8 $g l 1, \operatorname{ts}(3,3), \operatorname{td}(3,3,3,3), \operatorname{delt}(3,3), \operatorname{delt} 4(3,3,3,3)$

real*8 cc1, abbar

c

$\ll$

gentran(for $i: 1$ thru 3 do

(for $j: 1$ thru 3 do

$(t s[i, j]: \operatorname{abbar}(g l 1) * \operatorname{delt}[i, j]))) \$$

>>

$<$

gentran(for $i: 1$ thru 3 do

(for $j: 1$ thru 3 do

(for k:1 thru 3 do

(for $1: 1$ thru 3 do

$(\operatorname{td}[i, j, k, 1]: \operatorname{cc} 1(g 11) * \operatorname{delt} 4[i, j, k, 1]))))) \$$

\>

return

end

$\ll$

gentran (abbar (g11) :=block (type (function, abbar), type("real*8", abbar,gl1), abbar : eval (abbar))) \$

$\gg$

$\ll$

gentran (cc1 (g11):=block (type (function, cc1), type ("real*8", cc1,gl1), cc1:eval (cc1)))\$

>>

$<$

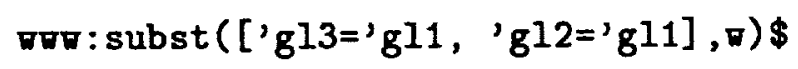

>> 
c

function sss1 (gl1)

<<cut (var); >>

$<$

gentran (type ("real*8", ss81, g11), sss1:2*eval (diff (rषv,' $g l 1,1))$ )\$

>

return

end

c

function sss11 (gl1)

<<cut (var); >>

$<<$

gentran(type("real*8", sss11,gl1), sss11:2*eval (diff(ww, 'gl1,2))) \$

\>

return

end 
Public reporting burden for this collection of information is estimated to average 1 hour per response, including the time for reviewing instructions, searching existing data sources, gathering and maintalning the data needed, and completing and reviewing the collection of information. Send comments regarding this burden estimate or any other aspect of this garlection of information, including sugestions for reducing this burden, to Washington Headquarters Services, Directorate for Information Operations and Reports, 1215 Jefferson Davis Highway, Sute 1204, Artington, VA 22202-4302, and to the Office of Management and Budget, Paperwork Reduction Project (0704-0188), Washington, DC 20503.

\begin{tabular}{|l|c|c|}
\hline 1. AGENCY USE ONLY (Leave blank) & $\begin{array}{c}\text { 2. REPORT DATE } \\
\text { April } 1993\end{array}$ & $\begin{array}{r}\text { 3. REPOAT TYPE AND DATES COVERED } \\
\text { Technical Memorandum }\end{array}$ \\
\hline
\end{tabular}

\section{TIRE AND SUBTITLE}

5. FUNDING NUMBERS

Explicit Robust Schemes for Implementation of General Principal

Value-Based Constitutive Models

6. AUTHOR(S)

WU-510-01-50

S.M. Arnold, A.F. Saleeb, H.Q. Tan, and Y. Zhang

\section{PERFORMING ORGANIZATION NAME(S) AND ADDRESS(ES)}

8. PERFORMING ORGANIZATION REPORT NUMBER

National Aeronautics and Space Administration

Lewis Research Center

E-7787

Cleveland, Ohio 44135-3191

9. SPONSORINGMONITORING AGENCY NAME(S) AND ADDRESS(ES)

10. SPONSORINGMONTORING AGENCY REPORT NUMBER

National Aeronautics and Space Administration

Washington, D.C. $20546-0001$

NASA TM-106123

\section{SUPPLEMENTARY NOTES}

S.M. Amold, NASA Lewis Research Center, Cleveland, Ohio; A.F. Saleeb, University of Akron, Department of Civil Engineering, Akron, Ohio 44325; and H.Q. Tan and Y. Zhang, University of Akron, Department of Mathematical Sciences, Akron, Ohio, 43325. Responsible person, S.M. Arnold, (216) 433-3334.

\begin{tabular}{|l|l} 
12a. DISTRIBUTION/AVAILABILIT STATEMENT & 12b. DISTRIBUTION CODE
\end{tabular}

Unclassified - Unlimited

Subject Category 459

\section{ABSTRACT (Maximum 200 words)}

The issue of developing effective and robust schemes to implement general hyperelastic constitutive models is addressed. To this end, special purpose functions are used to symbolically derive, evaluate, and automatically generate the associated FORTRAN code for the explicit forms of the corresponding stress function and material tangent stiffness tensors. These explicit forms are valid for the entire deformation range (i.e., with both distinct and repeated principal-stretch values). The analytical form of these explicit expressions is given here for the case in which the strain-energy potential $W$ is taken as a nonseparable polynomial function of the principle stretches.

14. SUBJECT TERMS

Hyperelastic; Constitutive models; Symbolic computation; Principal value

17. SECUATY CLASSIFICATION OF REPORT

Unclassified
18. SECURTY CLASSIFICATION OF THIS PAGE

- Unclassified
19. SECURTYY CLASSIFICATION OF ABSTRACT Unclassified
15. NUMBER OF PAGES 29

16. PRICE CODE $\mathrm{AO} 3$

20. LUITATION OF ABSTRACT

Standard Form 298 (Rev. 2-89) Prescribed by ANSI Std. Z39-18 298-102 